\section{Avaliação de um programa para promoção de hábitos alimentares saudáveis em escolares de Florianópolis, Santa Catarina, Brasil}

\section{Evaluation of a program to promote healthy eating habits among schoolchildren in the city of Florianópolis, State of Santa Catarina, Brazil}

Cristine Garcia Gabriel 1

Melina Valério dos Santos 2

Francisco de Assis Guedes de Vasconcelos 3

1-3 Programa de Pós-Graduação em Nutrição. Departamento de Nutrição. Centro de Ciências da Saúde, Universidade Federal de Santa Catarina. Trindade. Florianópolis, Santa Catarina, Brasil. CEP: 88.040-970. E-mail: cris_ntr@hotmail.com

\begin{abstract}
Objectives: to describe the results of a nutrition intervention program aiming to promote healthy eating habits among schoolchildren.

Methods: an uncontrolled intervention study was conducted covering 162 schoolchildren from the 3 rd and 4 th grades of two educational institutions (public and private) in the city of Florianópolis, Santa Catarina, Brazil. A short-term nutritional education program was run over the course of seven weekly meetings. An eating habits questionnaire was applied. Weight and height data were taken before and one month after the intervention conclusion.

Results: although no changes were detected in the schoolchildren nutritional profile, there was a significant reduction in the cookies consumption brought from home among private school boys and in the public school, there was a significant increase in the school meals and fruit consumption.

Conclusions: after the intervention, it was observed that it had increased the frequency of some attitudes and more healthful eating habits. The period between the program conclusion and the second anthropometric examination can not have been enough to provoke changes in the body mass index. This experience can be applied in other institutions and, for its better effectiveness, it must count with the school community participation (mainly parents and professors).
\end{abstract}

Key words School feeding, Overweight, Obesity, Nutrition intervention, Food habits

\section{Resumo}

Objetivos: avaliar os resultados de um programa de intervenção nutricional visando à promoção de hábitos alimentares saudáveis em escolares do ensino fundamental.

Métodos: estudo de intervenção não controlado desenvolvido com 162 escolares de terceira e quarta séries de duas instituições de ensino (pública e privada), em Florianópolis, Santa Catarina, Brasil. O programa de educação nutricional de curta duração foi distribuído em sete encontros. Aplicou-se um questionário de consumo alimentar e aferiu-se peso e estatura antes e um mês após o término da intervenção.

Resultados: embora não tenham sido detectadas mudanças no perfil nutricional dos escolares, percebeuse na escola privada redução significativa nos percentuais de bolachas recheadas trazidas de casa pelos meninos e na escola pública aumentou significativamente o consumo da merenda escolar e aceitação por frutas.

Conclusões: após a intervenção, verificou-se aumento da freqüencia de algumas atitudes e práticas alimentares mais saudáveis. O tempo entre a conclusão do programa e a realização do segundo exame antropométrico pode não ter sido suficiente para provocar mudanças no índice de massa corporal. Essa experiência pode ser aplicada em outras instituições e, para sua maior efetividade, deve contar com a participação da comunidade escolar (especialmente pais $e$ professores).

Palavras-chave Alimentação escolar, Sobrepeso, Obesidade, Intervenção nutricional, Hábitos alimentares 


\section{Introdução}

A obesidade tem constituído a doença crônica não transmissível de maior prevalência nas sociedades industrializadas. ${ }^{1,2}$ Sua presença em idades cada vez mais precoces tem preocupado e desafiado pesquisadores e profissionais da saúde. ${ }^{2-5}$

Devido à dificuldade na obtenção de sucesso no tratamento da obesidade em adultos, torna-se necessário a implementação de medidas de prevenção e tratamento desse distúrbio nutricional ainda na infância. ${ }^{6}$ Nessa fase, além de tratar a obesidade, deve-se buscar a incorporação de um estilo de vida saudável de maneira gradual e duradoura, com ênfase na formação de hábitos alimentares adequados e na prática de atividades físicas. ${ }^{7}$

Estudo realizado por Assis et al.,8 em uma amostra representativa de escolares de Florianópolis, Santa Catarina, constatou altas prevalências de sobrepeso e obesidade infantil, de acordo com critério de Must et al. 9 Frente a esses resultados, que foram concordantes com estudos previamente realizados em outros países em desenvolvimento, 10-12 os autores recomendaram a instituição imediata de estratégias de invtervenção voltadas à promoção de hábitos alimentares saudáveis e de incentivo à prática de atividades físicas nas escolas de ensino fundamental do minicípio

Um bom argumento para a implementação de programas preventivos é o seu custo reduzido quando comparado aos gastos com o tratamento das patologias associadas à obesidade.13 Entre as possíveis medidas de intervenção, destaca-se a educação nutricional como um componente decisivo na promoção de saúde.7,14 Embora não restem dúvidas sobre sua importância, as reflexões sobre as possibilidades e limites da educação alimentar e nutricional, assim como o modo como ela é concebida, ainda são escassas. 15

As intervenções nutricionais direcionadas à obesidade desenvolvem-se, com maior freqüência, na prática clínica, todavia, sabe-se que as escolas também propiciam uma oportunidade valiosa para prevenir e tratar esse distúrbio. ${ }^{16}$

A American Dietetic Association (ADA), a Society for Nutrition Education, e a American School Food Service Association (ASFSA) ressaltam a importância de a escola desenvolver estratégias de intervenção que envolvam toda a comunidade escolar na formação de hábitos de vida saudáveis, propiciando aos escolares um ambiente com opções de lanches nutricionalmente equilibrados, exercícios físicos regulares e programas de educação nutricional. 17
Programas educativos direcionados a escolares constituem uma das estratégias mais importantes para enfrentar a obesidade infantil, já que a cobertura no ensino fundamental é de praticamente $100 \% \cdot{ }^{13,18,19} \mathrm{O}$ contexto desafiador da educação nutricional exige o desenvolvimento de abordagens que permitam abraçar os problemas alimentares de modo mais amplo, por intermédio de estratégias que superem a mera transmissão de informações. ${ }^{20,21}$

Este artigo relata os resultados de um programa de intervenção nutricional visando à promoção de hábitos alimentares saudáveis em escolares do ensino fundamental.

\section{Métodos}

Esta pesquisa incluiu todos os escolares matriculados nas terceira e quarta séries da Escola Básica José Boiteux (escola pública estadual) e nas terceira séries do Colégio Nossa Senhora de Fátima (escola privada), já que as quarta séries dessa instituição estavam participando de outros projetos no período em que se desenvolveu este estudo (fevereiro a julho de 2004). Ambas as escolas localizam-se na região continental do município de Florianópolis, Santa Catarina, Brasil, e foram escolhidas de forma intencional, entre 16 instituições participantes de investigação anterior. 9 Os critérios para escolha das instituições incluíram a existência de cantina escolar, a aceitação por parte da direção e o fato de localizarem-se no mesmo bairro, facilitando a operacionalização da investigação.

Ressalta-se, todavia, que essa pesquisa incluiu somente as terceira e quarta séries, uma vez que se elaborou um programa de atividades de intervenção nutricional específico para o nível cognitivo esperado em escolares destas séries ou faixa etária.

As informações sobre o consumo alimentar foram obtidas mediante a utilização de um questionário sobre o consumo de alimentos no período de permanência na escola. Salienta-se que os alunos da escola privada poderiam referir o consumo na escola de alimentos adquiridos na cantina e/ou lanches trazidos de casa, enquanto os alunos da escola pública, além dessas modalidades, poderiam relatar também o consumo de alimentos fornecidos pelo Programa Nacional de Alimentação Escolar (PNAE).

Deste modo, o referido questionário foi composto por questões abertas e fechadas contidas em duas partes distintas (bloco A e bloco B). O bloco A, específico para os alunos da escola pública, foi estruturado com questões relativas ao consumo da merenda escolar, oferecida pelo PNAE. O bloco B, 
destinado a todos os escolares, constituiu-se de questões referentes às práticas de trazer lanche de casa e comprar alimentos na cantina. Acadêmicas de graduação em Nutrição da Universidade Federal de Santa Catarina (UFSC) entrevistaram os escolares, após processo de treinamento e realização de préteste.

Assim, as variáveis relativas ao consumo alimentar na escola, utilizadas para avaliar o efeito da intervenção nutricional foram: os alimentos mais adquiridos nas cantinas; os alimentos que, de acordo com a preferência dos escolares, deveriam ser comercializados nas cantinas; os alimentos mais trazidos de casa; o consumo ou não da merenda escolar; os alimentos preferidos na merenda escolar e, os alimentos que deveriam ser oferecidos pelo PNAE, de acordo com a opinião dos escolares. Tais dados foram coletados com os mesmos escolares em dois momentos: antes e após a intervenção educativa, com intervalo de quatro meses entre as coletas (nos meses de março e agosto de 2004, respectivamente).

Para a avaliação nutricional dos escolares, obteve-se informações sobre peso, estatura, idade e sexo, de acordo com os protocolos para coleta de medidas antropométricas recomendados pela Organização Mundial de Saúde.22 O peso dos sujeitos foi aferido por meio de uma balança digital, marca Marte ${ }^{\circledR}$, com precisão de $0,05 \mathrm{~kg}$. Para medir a estatura, utilizou-se fita métrica milimetrada, fixada à parede, com o ponto zero no nível do solo e esquadro. Para tanto, as crianças estavam sem sapatos, em posição ortostática, com a massa do corpo distribuída nas duas pernas, braços ao longo do corpo, pés unidos, joelhos esticados e a cabeça orientada no plano horizontal de Frankfurt. A medida foi obtida em apnéia após uma inspiração profunda.

Calculou-se o Índice de Massa Corporal (IMC), em $\mathrm{kg} / \mathrm{m}^{2}$, de cada escolar, e determinou-se o perfil nutricional da população através do critério recomendado pelo Centers for Disease Control and Prevention (CDC). 23 Por meio deste critério, os escolares foram classificados nas seguintes categorias de estado nutricional: sobrepeso e obesidade, eutrofia e baixo peso. Deste modo, para avaliar o efeito da intervenção sobre o estado nutricional, utilizaram-se como variáveis tais categorias, sendo estas registradas também nos dois momentos de coleta de dados com os escolares, antes e após a intervenção educativa (março e agosto de 2004).

Pela comparação dessas variáveis e das variáveis relativas ao consumo alimentar, descritas anteriormente, pode-se avaliar o efeito da intervenção educativa, segundo o sexo dos escolares (meninos e meninas) e tipo de escola (pública e privada).

As crianças que estavam ausentes nos dias de coleta de dados, foram consideradas não resposta após três dias de tentativa.

O planejamento das atividades educativas passou por processo de aprimoramento, contando-se com a assessoria de distintos profissionais, particularmente, de nutricionista e pedagoga da Secretaria Municipal de Educação de Florianópolis. Delineouse um programa de curta duração com sete encontros, sendo um por semana. O primeiro relacionouse à integração dos participantes; o segundo abordou o Guia da Pirâmide Alimentar;24 o terceiro deu ênfase aos nutrientes específicos e suas funções; no quarto, compararam-se as calorias de alimentos pouco e muito nutritivos; no quinto, desenvolveu-se um jogo visando avaliar os conhecimentos adquiridos; o sexto trabalhou a separação e a reciclagem do lixo, a partir de embalagens de alimentos jogadas pelos escolares; e o último, o qual abrangeu todos os conteúdos, abordou a montagem de um cardápio com seis refeições diárias.

O programa de educação nutricional foi desenvolvido ao longo do primeiro semestre letivo de 2004, enquanto a coleta dos dados antropométricos e de consumo alimentar ocorreu em dois momentos: antes e um mês após o término da intervenção educativa.

Os dados foram processados e analisados usando-se os programas Microsoft Excel e Statistical Package for Social Sciences (SPSS) versão 8.0. A análise descritiva foi feita a partir de medidas de dispersão e tendência central por distribuição de freqüência. Utilizou-se o teste estatístico qui-quadrado $(\chi 2)$ para as variáveis categóricas, considerado significativo quando o valor de $p$ for menor que 0,05 .

O protocolo da pesquisa foi aprovado pelo Comitê de Ética da Universidade Federal de Santa Catarina, de acordo com as normas estabelecidas pela Resolução 196/96 do Conselho Nacional de Saúde.

\section{Resultados}

Dos 214 escolares matriculados em 2004 nas terceira e quarta séries do ensino fundamental da instituição pública e nas terceira séries da escola privada, 178 $(83,2 \%)$ participaram da primeira coleta de dados e, por conseguinte, do programa de intervenção. Dentre os $36(16,8 \%)$ escolares que não participaram, 9 escolares obtiveram recusa por parte dos pais $(5 \mathrm{da}$ rede pública e 4 da privada) e 27 escolares não foram encontrados devido à ausência nos dias de coleta de 
Tabela 1

Distribuição absoluta e percentual do diagnóstico nutricional dos escolares investigados, antes e após a intervenção nutricional, por sexo e natureza da escola. Florianópolis, Santa Catarina, 2004.

\begin{tabular}{|c|c|c|c|c|c|c|c|c|c|c|c|c|c|c|c|}
\hline \multicolumn{16}{|c|}{ Diagnóstico nutricional } \\
\hline & \multicolumn{4}{|c|}{ Sobrepeso e obesidade } & \multirow{3}{*}{$p$} & \multicolumn{4}{|c|}{ Eutrofia } & \multicolumn{6}{|c|}{ Baixo peso } \\
\hline & \multicolumn{2}{|c|}{ Antes } & \multicolumn{2}{|c|}{ Após } & & \multicolumn{2}{|c|}{ Antes } & \multicolumn{2}{|c|}{ Após } & \multirow[t]{2}{*}{$p$} & \multicolumn{2}{|c|}{ Antes } & \multicolumn{2}{|c|}{ Após } & \multirow[t]{2}{*}{$p$} \\
\hline & $\mathrm{n}$ & $\%$ & $\mathrm{n}$ & $\%$ & & $\mathrm{n}$ & $\%$ & $\mathrm{n}$ & $\%$ & & $\mathrm{n}$ & $\%$ & $\mathrm{n}$ & $\%$ & \\
\hline \multicolumn{16}{|l|}{ Sexo } \\
\hline Meninos $(n=73)$ & 20 & 27,5 & 20 & 27,4 & 0,852 & 51 & 70,0 & 52 & 71,2 & 1,000 & 2 & 2,5 & 1 & 1,4 & 0,500 \\
\hline Meninas $(n=89)$ & 15 & 16,3 & 9 & 10,1 & 0,572 & 69 & 77,6 & 73 & 82,1 & 0,575 & 5 & 6,1 & 7 & 7,8 & 0,764 \\
\hline Total $(n=162)$ & 36 & 21,9 & 29 & 17,9 & 0,405 & 119 & 73,6 & 12 & 77,2 & 0,519 & 7 & 4,5 & 8 & 4,9 & 0,764 \\
\hline \multicolumn{16}{|l|}{ Escola } \\
\hline Pública $(\mathrm{n}=85)$ & 17 & 20,2 & 18 & 21,2 & 1,000 & 62 & 72,7 & 62 & 72,9 & 0,862 & 6 & 7,1 & 5 & 5,9 & 1,000 \\
\hline Privada $(n=77)$ & 18 & 24,0 & 11 & 14,3 & 0,216 & 58 & 74,7 & 63 & 81,8 & 0,432 & 1 & 1,3 & 3 & 3,9 & 0,310 \\
\hline
\end{tabular}

dados (18 da rede pública e 9 da privada). Na segunda coleta, $162(91,0 \%)$ dos 178 escolares que haviam participado da primeira coleta, foram entrevistados. Dezesseis escolares $(9,0 \%)$ não foram encontrados devido a faltas e transferências. Entre os escolares que compuseram esta amostra, 52,5\% eram da escola pública e $47,5 \%$ da escola privada, sendo $45,1 \%$ do sexo masculino e $54,9 \%$ do sexo feminino. A média da idade dos meninos e meninas variou entre $9,0(0,45)$ e $9,1(0,53)$ anos, respectivamente.

Em relação à avaliação antropométrica, os resultados apontaram uma prevalência geral de obesidade/sobrepeso de $21,9 \%$ antes do programa de intervenção e 17,9\% após o mesmo. A intervenção nutricional não modificou o perfil nutricional dos escolares, uma vez que não houve diferenças significativas nas prevalências totais, por sexo e/ou por instituição antes e após o programa (Tabela 1).

$\mathrm{Na}$ escola pública, as mini-pizzas destacaram-se como a preparação mais adquirida pelos escolares de ambos os sexos nas cantinas, antes (meninos: $38,1 \%$; meninas: $34,6 \%$ ) e após (meninos: $29,7 \%$; meninas: $31,8 \%$ ) o desenvolvimento do programa educativo. Não foram encontradas diferenças significativas entre os alimentos mais adquiridos, nos dois momentos da pesquisa, em ambas as instituições.

Com relação aos alimentos que deveriam ser comercializados nas cantinas, de acordo com a preferência dos escolares, observa-se na Tabela 2 que, antes da intervenção nutricional, os refrige- rantes, seguidos por picolés/sorvetes, foram os alimentos mais citados pelos meninos da escola pública. As preferências entre as meninas dessa rede de ensino foram os salgados fritos e salgadinhos tipo chips. Após a realização do programa, os sorvetes/picolés não foram citados pelos meninos, que demonstraram preferência por sucos naturais de frutas e cachorro quente. Porém, as meninas não apresentaram mudanças que demonstrassem hábitos mais saudáveis.

As frutas foram citadas pelos meninos da escola privada, antes e após a intervenção nutricional (30,0\% e $28,7 \%$, respectivamente), como alimentos que deveriam ser vendidos nas cantinas. Os meninos da escola pública não citaram frutas em nenhum dos momentos (Tabela 2). As balas/chicletes foram também bastante citadas pelos meninos da rede privada, no primeiro momento $(20,0 \%)$, porém, após o trabalho de educação nutricional, não se observou nenhuma referência a essas guloseimas. Os meninos da rede pública também não referiram estes alimentos.

Comparando-se as preferências das meninas de ambas as instituições, observa-se que os salgadinhos tipo chips, salgados fritos, sorvetes/picolés e refrigerantes estão mais presentes na escola pública, mesmo após a intervenção. As meninas da escola privada não citaram refrigerantes e as mini-pizzas após o desenvolvimento do programa, entretanto sobressaíram-se quanto à preferência por balas/chicletes (privada: 35,8\% e pública: 8,9\%; 
Tabela 2

Alimentos que deveriam ser vendidos nas cantinas de acordo com a preferência dos alunos, antes e após a intervenção nutricional, segundo o sexo. Florianópolis, Santa Catarina, 2004.

\begin{tabular}{|c|c|c|c|c|c|c|c|c|c|c|}
\hline \multirow[t]{3}{*}{ Alimentos } & \multicolumn{4}{|c|}{ Meninos } & \multicolumn{6}{|c|}{ Meninas } \\
\hline & \multicolumn{2}{|c|}{ Antes } & \multicolumn{2}{|c|}{ Após } & \multirow[b]{2}{*}{$p$} & \multicolumn{2}{|c|}{ Antes } & \multicolumn{2}{|c|}{ Após } & \multirow[b]{2}{*}{$p$} \\
\hline & $\mathrm{n}$ & $\%$ & $\mathrm{n}$ & $\%$ & & $\mathrm{n}$ & $\%$ & $\mathrm{n}$ & $\%$ & \\
\hline \multicolumn{11}{|l|}{ Escola Pública } \\
\hline Refrigerantes & 8 & 22,8 & 3 & 7,7 & 0,132 & 11 & 10,9 & 9 & 20,0 & 0,262 \\
\hline Sorvetes/Picolés & 6 & 18,2 & - & - & - & 5 & 5,4 & 3 & 6,8 & 0,492 \\
\hline Biscoitos & 3 & 9,1 & 3 & 7,7 & 0,609 & - & - & 1 & 2,2 & - \\
\hline Bolos & 3 & 9,1 & 3 & 7,7 & - & - & - & 1 & 2,2 & - \\
\hline Salgadinhos tipo chips & 3 & 9,1 & 3 & 7,7 & 0,609 & 17 & 16,4 & 10 & 22,2 & 0,664 \\
\hline Salgados fritos & 3 & 9,1 & 3 & 7,7 & 0,609 & 18 & 18,2 & 7 & 15,7 & 0,841 \\
\hline Sucos naturais & 3 & 9,1 & 6 & 15,4 & 0,297 & 7 & 7,3 & 2 & 4,4 & 0,413 \\
\hline logurtes & 2 & 4,5 & - & - & - & - & - & - & - & - \\
\hline Cachorro quente & 2 & 4,5 & 6 & 15,4 & 0,168 & 5 & 5,4 & 2 & 4,4 & 0,609 \\
\hline Balas/Chicletes & 2 & 4,5 & 3 & 7,7 & 0,552 & 9 & 9,1 & 4 & $8,9 \% \mathrm{~b}$ & 0,605 \\
\hline Frutas & - & - & - & - & - & 10 & 10,9 & - & - & - \\
\hline Torrada & - & - & - & - & - & 5 & 5,4 & - & - & - \\
\hline Doces & - & - & 3 & 7,7 & - & 3 & 3,6 & - & - & - \\
\hline Bolachas recheadas & - & - & 3 & 7,7 & - & - & - & - & - & - \\
\hline Hambúrguer & - & - & 3 & 7,7 & - & - & - & - & - & - \\
\hline Salada de frutas & - & - & - & - & - & - & - & 2 & 4,4 & - \\
\hline Salgados assados & - & - & 3 & 7,7 & - & - & - & - & - & - \\
\hline Chocolates & - & - & - & - & - & - & - & 2 & 4,4 & - \\
\hline Outros & - & - & - & - & - & 7 & 7,4 & 2 & 4,4 & 0,413 \\
\hline \multicolumn{11}{|l|}{ Escola Privada } \\
\hline Frutas & 3 & 30,0 & 4 & 28,7 & 0,643 & 2 & 14,3 & 1 & 7,1 & 0,500 \\
\hline Balas/chicletes & 2 & 20,0 & - & - & - & - & - & 5 & $35,8 \mathrm{~b}$ & - \\
\hline Salgados fritos & 1 & 10,0 & 3 & 21,5 & 0,436 & - & - & 1 & 7,1 & - \\
\hline Hambúrguer & 1 & 10,0 & - & - & - & - & - & - & - & - \\
\hline logurtes & 1 & 10,0 & - & - & - & - & - & - & - & - \\
\hline Refrigerantes & 1 & 10,0 & 2 & $14,3 \%$ & 0,629 & 2 & 14,3 & & & - \\
\hline Sanduíches & 1 & 10,0 & - & - & - & - & - & 4 & 28,6 & - \\
\hline Sucos naturais & - & - & 1 & $7,1 \%$ & - & 2 & 14,3 & 2 & $14,3 a$ & 0,702 \\
\hline Salgadinhos tipo Chips & - & - & 1 & $7,1 \%$ & - & - & - & - & - & - \\
\hline Doces & - & - & 1 & $7,1 \%$ & - & - & - & - & - & - \\
\hline Bolos & - & - & 1 & $7,1 \%$ & - & - & - & - & - & - \\
\hline Salgados assados & - & - & - & - & - & 4 & 28,5 & - & - & - \\
\hline Mini pizza & - & - & - & - & - & 2 & 14,3 & - & - & - \\
\hline Pão de queijo & - & - & - & - & - & 2 & 14,3 & - & - & - \\
\hline Chá & & - & - & - & - & - & - & 1 & 7,1 & - \\
\hline Outros & & & 1 & $7,1 \%$ & - & - & - & - & - & - \\
\hline
\end{tabular}

a,b Apresentaram diferença estatística $p<0,05)$; a $p=0,024 ;$ b $p=0,003$.

$p=0,003)$ e sucos naturais (privada: $14,3 \%$ e pública: $4,4 \% p=0,024)$. (Tabela 2 )

Os alunos também foram questionados sobre os alimentos trazidos de casa (Tabela 3). Embora na escola pública, após a intervenção, tenha se observado uma diminuição de bolachas recheadas (antes: $24,6 \%$ e após: $16,5 \%$ ) e sucos artificiais (antes: $17,4 \%$ e após: $10,1 \%$ ) e aumento de frutas (antes: 
Tabela 3

Alimentos trazidos de casa pelos alunos antes e após a intervenção nutricional, segundo o sexo. Florianópolis, Santa Catarina, 2004.

\begin{tabular}{|c|c|c|c|c|c|c|c|c|c|c|}
\hline \multirow[t]{3}{*}{ Alimentos } & \multicolumn{4}{|c|}{ Meninos } & \multicolumn{6}{|c|}{ Meninas } \\
\hline & \multicolumn{2}{|c|}{ Antes } & \multicolumn{2}{|c|}{ Após } & \multirow[b]{2}{*}{$p$} & \multicolumn{2}{|c|}{ Antes } & \multicolumn{2}{|c|}{ Após } & \multirow[b]{2}{*}{$p$} \\
\hline & $\mathrm{n}$ & $\%$ & $\mathrm{n}$ & $\%$ & & $\mathrm{n}$ & $\%$ & $\mathrm{n}$ & $\%$ & \\
\hline \multicolumn{11}{|l|}{ Escola Pública } \\
\hline Bolachas recheadas & 37 & 24,6 & 33 & 16,5 & 0,079 & 43 & 20,0 & 54 & 22,6 & 0,576 \\
\hline Sucos artificiais & 26 & 17,4 & 20 & 10,1 & 0,064 & 35 & 16,4 & 24 & 10,2 & 0,066 \\
\hline Frutas & 18 & 11,6 & 33 & 16,5 & 0,304 & 9 & 4,3 & 12 & 5,1 & 0,842 \\
\hline Biscoitos & 18 & 11,6 & 24 & 12,7 & 0,868 & 29 & 13,5 & 19 & 8,0 & 0,077 \\
\hline Salgadinhos tipo chips & 13 & 8,7 & 13 & 6,3 & 0,576 & 32 & 15,0 & 31 & 13,1 & 0,650 \\
\hline Sanduíches & 13 & 8,7 & 24 & 12,7 & 0,407 & 6 & 2,9 & 12 & 5,1 & 0,329 \\
\hline Sucos naturais & 6 & 4,3 & 13 & 6,3 & 0,433 & 9 & $4,3 a$ & 23 & $9,5 a$ & 0,037 \\
\hline Refrigerantes & 4 & 2,9 & 7 & 3,8 & 0,453 & 22 & 10,0 & 24 & 10,2 & 0,929 \\
\hline Pães & 4 & 2,9 & 3 & 1,3 & 0,345 & - & & 9 & 3,6 & - \\
\hline Achocolatados & - & - & 18 & 8,9 & - & 6 & 2,9 & 7 & 2,9 & 0,846 \\
\hline Chocolates & - & - & 3 & 1,3 & - & 5 & 2,1 & - & & - \\
\hline Bolos & - & - & 3 & 1,3 & - & - & - & 11 & 4,4 & - \\
\hline logurtes & - & - & 3 & 1,3 & - & - & - & 4 & 1,5 & - \\
\hline Outros & 11 & $7,3 b$ & 3 & $1,0^{b}$ & 0,013 & 19 & $8,6 c$ & 9 & $3,8 \mathrm{c}$ & 0,040 \\
\hline \multicolumn{11}{|l|}{ Escola Privada } \\
\hline Bolachas recheadas & 17 & $22,7 d$ & 7 & $10,1 d$ & 0,043 & 24 & 26,4 & 17 & 15,6 & 0,088 \\
\hline Sucos artificiais & 11 & 14,7 & 10 & 14,5 & 0,836 & 5 & 5,5 & 8 & 7,3 & 0,811 \\
\hline Sanduíches & 9 & 12,0 & 7 & 10,1 & 0,929 & 10 & 10,9 & 13 & 11,9 & 0,987 \\
\hline Frutas & 7 & 9,3 & 2 & 2,9 & 0,104 & 11 & 11,8 & 13 & 11,9 & 0,854 \\
\hline Biscoitos & 7 & 9,3 & 11 & 15,9 & 0,344 & 8 & 9,1 & 17 & 15,6 & 0,217 \\
\hline Achocolatados & 7 & 9,3 & - & - & - & 6 & 6,4 & 4 & 3,7 & 0,267 \\
\hline Sucos naturais & 4 & 5,3 & 7 & 10,1 & 0,440 & 3 & 3,6 & 9 & 8,3 & 0,241 \\
\hline Barra de cereais & 2 & 2,7 & 3 & 4,3 & 0,460 & - & - & - & & - \\
\hline logurtes & 2 & 2,7 & 3 & 4,3 & 0,460 & - & - & 4 & 3,7 & - \\
\hline Pão de queijo & 2 & 2,7 & - & - & - & - & - & - & - & - \\
\hline Pães & - & - & 8 & 11,6 & - & - & - & 7 & 6,4 & - \\
\hline Bolos & - & - & 5 & 7,3 & - & 7 & 8,2 & 11 & 10,1 & 0,732 \\
\hline Salgadinhos tipo chips & - & - & - & - & - & 5 & 5,5 & - & - & - \\
\hline Outros & 7 & 9,3 & 6 & 8,9 & 0,874 & 12 & 12,6 & 6 & 5,5 & 0,100 \\
\hline
\end{tabular}

a,b,c,d Apresentaram diferença estatística $p<0,05)$.

11,6\% e após: 16,5\%), sanduíches (antes: 8,7\% e após: $12,7 \%$ ) e achocolatados (antes: $0 \%$ e após: $8,9 \%$ ) por parte dos meninos, esses valores não foram significativos. Quanto às meninas dessa instituição, o percentual de sucos artificiais (antes: $16,4 \%$ e após: $10,2 \%$ ) e biscoitos (antes: $13,5 \%$ e após: $8,0 \%$ ) reduziu na segunda coleta, porém sem diferenças significativas. Já com relação aos sucos naturais, ressalta-se que estes aumentaram significativamente após a intervenção (antes: $4,3 \%$ e após: $9,5 \% ; p=0,037$ ). (Tabela 3 )
Já na escola privada, percebeu-se uma redução nos percentuais referentes às bolachas recheadas após o programa, mostrando diferença significativa para os meninos (antes: $22,7 \%$ e após: 10,1\%; $p=0,043$ ). (Tabela 3)

Comparando-se os lanches trazidos de casa antes das ações educativas por ambas as instituições, sem distinção de sexo, constatou-se que os salgadinhos tipo chips e os sucos artificiais sobressaíam-se na escola pública ( $12,3 \%$ e $14,0 \%$, respectivamente), quando comparada à rede privada $(3,0 \%$ e $9,0 \%$, 
Alimentos preferidos e alimentos que deveriam ser oferecidos (sugeridos) pelo Programa Nacional de Alimentação Escolar (PNAE), de acordo com os alunos entrevistados na escola pública, antes e após a intervenção nutricional, segundo o sexo. Florianópolis, Santa Catarina, 2004.

\begin{tabular}{|c|c|c|c|c|c|c|c|c|c|c|}
\hline \multirow[t]{3}{*}{ Alimentos } & \multicolumn{4}{|c|}{ Meninos } & \multicolumn{6}{|c|}{ Meninas } \\
\hline & \multicolumn{2}{|c|}{ Antes } & \multicolumn{2}{|c|}{ Após } & \multirow[b]{2}{*}{$p$} & \multicolumn{2}{|c|}{ Antes } & \multicolumn{2}{|c|}{ Após } & \multirow[b]{2}{*}{$p$} \\
\hline & $\mathrm{n}$ & $\%$ & $\mathrm{n}$ & $\%$ & & $\mathrm{n}$ & $\%$ & $\mathrm{n}$ & $\%$ & \\
\hline \multicolumn{11}{|l|}{ Preferidos } \\
\hline Biscoitos & 36 & $31,1 \mathrm{a}$ & 21 & $13,9 a$ & 0,001 & 25 & 20,2 & 20 & 12,2 & 0,093 \\
\hline Achocolatados & 15 & $13,1 \mathrm{~b}$ & 7 & $4,6 \mathrm{~b}$ & 0,025 & 13 & $10,8 d$ & 6 & $3,7 \mathrm{~d}$ & 0,038 \\
\hline Arroz e feijão & 15 & 13,1 & 19 & 12,3 & 0,931 & 7 & 5,4 & 20 & 12,2 & 0,092 \\
\hline Sucrilhos & 11 & 9,8 & 14 & 9,2 & 0,887 & 22 & $17,6 \mathrm{e}$ & 14 & $8,5 \mathrm{e}$ & 0,030 \\
\hline Sopas/canjas & 8 & 6,6 & 19 & 12,3 & 0,190 & 20 & 16,2 & 28 & 17,1 & 0,957 \\
\hline Mingau & 6 & 4,9 & 7 & 4,6 & 0,939 & 7 & 5,4 & 16 & 9,8 & 0,291 \\
\hline Sucos & 6 & 4,9 & - & & - & 5 & 4,1 & - & - & - \\
\hline Macarrão & 6 & 4,9 & - & & - & 5 & 4,1 & 4 & 2,4 & 0,331 \\
\hline Carnes & 4 & 3,3 & 7 & 4,6 & 0,439 & 5 & 4,1 & 16 & 9,8 & 0,105 \\
\hline Frutas & 2 & $1,7 \mathrm{c}$ & 35 & $23,1 \mathrm{c}$ & 0,001 & 5 & $4,1^{f}$ & 24 & $14,6^{f}$ & 0,005 \\
\hline Pão com doce & - & - & 12 & 7,7 & - & - & - & 4 & 2,4 & - \\
\hline Leite/iogurte & - & - & - & - & - & - & - & 4 & 2,4 & - \\
\hline Cachorro quente & - & - & 5 & 3,1 & - & - & - & - & - & - \\
\hline Outros & 8 & 6,6 & 7 & 4,6 & 0,591 & 10 & 8,0 & 8 & 4,9 & 0,389 \\
\hline \multicolumn{11}{|l|}{ Sugeridos } \\
\hline Frutas & 14 & 20,6 & 20 & 10,4 & 0,053 & - & - & 17 & 15,2 & - \\
\hline Arroz e feijão & $6 g$ & 8,8 & 4 & 2,19 & 0,022 & - & - & - & - & - \\
\hline Cachorro quente & 6 & 8,8 & 12 & 6,2 & 0,659 & - & - & 5 & 4,5 & - \\
\hline Doces & 6 & 8,8 & 8 & 4,2 & 0,250 & 4 & 4,0 & - & & - \\
\hline Sucos naturais & 4 & 5,9 & 12 & 6,2 & 0,588 & 10 & 10,0 & 8 & 7,6 & 0,661 \\
\hline Pizzas & 4 & 5,9 & 12 & 6,2 & 0,588 & 4 & 4,0 & - & - & - \\
\hline Carnes & 4 & 5,9 & 20 & 10,4 & 0,386 & 4 & 4,0 & 8 & 7,6 & 0,459 \\
\hline Refrigerantes & 4 & 5,9 & 4 & 2,1 & 0,126 & - & & - & - & - \\
\hline Bolachas recheadas & 4 & 5,9 & 16 & 8,4 & 0,698 & 10 & 10,0 & 10 & 9,1 & 0,973 \\
\hline logurtes & 4 & 5,9 & - & & - & 20 & $20,0 \mathrm{~h}$ & 3 & $3,0 \mathrm{~h}$ & 0,001 \\
\hline Preparações fritas & 2 & 2,9 & 20 & 10,4 & 0,098 & 4 & 4,0 & 5 & 4,5 & 0,553 \\
\hline Salgadinhos tipo chips & 2 & 2,9 & 4 & 2,1 & 0,494 & 6 & 6,0 & 8 & 7,6 & 0,912 \\
\hline Macarrão & - & - & 4 & 2,1 & - & 8 & 8,0 & 7 & 6,1 & 0,862 \\
\hline Bolos & - & - & - & & - & 8 & 8,0 & - & & - \\
\hline Chocolates & - & - & - & - & - & 4 & 4,0 & 3 & 3,0 & 0,452 \\
\hline Balas/Chicletes & - & - & - & & - & 6 & 6,0 & 3 & 3,0 & 0,208 \\
\hline Biscoitos & - & - & 8 & 4,2 & - & - & - & 7 & 6,1 & - \\
\hline Achocolatados & - & - & 8 & 4,2 & - & - & - & 5 & 4,5 & - \\
\hline Sucrilhos & - & & 4 & 2,1 & - & - & - & 5 & 4,5 & - \\
\hline Sanduíches & - & - & 12 & 6,2 & - & - & - & 3 & 3,0 & - \\
\hline Salgados assados & & & 12 & 6,2 & - & - & & - & & - \\
\hline Outros & 8 & 11,8 & - & - & 0,229 & 12 & 12,0 & 12 & 10,7 & 0,994 \\
\hline
\end{tabular}

a,b,c,d,e,f,g,h Apresentaram diferença estatística $p<0,05)$.

respectivamente) $(p=0,001$ e $p=0,044)$. Os salgadinhos continuaram elevados nessa instituição mesmo após o programa, não aparecendo mais na escola privada. Os refrigerantes não são citados na instituição privada em nenhum dos momentos, ao contrário do que ocorreu na escola pública.

Especificamente à escola pública, o presente estudo detectou ainda um aumento no consumo da 
merenda escolar oferecida de 59,4\% ( $\mathrm{n}=50$ escolares) antes da intervenção nutricional para 74,4\% $(\mathrm{n}=63$ escolares) após a mesma $(p=0,034)$. Nos dois momentos de coleta de dados, não foram encontradas diferenças estatisticamente significantes entre os sexos.

Os escolares foram questionados a respeito de suas preferências entre os alimentos que fazem parte dos cardápios do PNAE. Os biscoitos foram os alimentos mais citados por escolares de ambos os sexos, antes da intervenção nutricional. Entre as preferências das meninas estavam também sucrilhos e canjas/sopas, respectivamente. Além dos biscoitos, faziam parte das preferências dos meninos, nesse primeiro momento, achocolatados e arroz com feijão. (Tabela 4)

$\mathrm{Na}$ escola pública, após a intervenção nutricional, a aceitação por frutas aumentou significativamente para as crianças dos sexos masculino (antes: $1,7 \%$ e após: $23,1 \% ; p=0,001)$ e feminino (antes: $4,1 \%$ e após: $14,6 \% ; p=0,005)$, passando a ocupar o primeiro lugar na lista de alimentos preferidos entre os meninos. Ainda entre os garotos, verificou-se que a preferência por biscoitos diminuiu significativamente (antes: $31,1 \%$ e após: $13,9 \% ; p=0,001$ ), assim como por achocolatados (antes: $13,1 \%$ e após: 4,6\%; $p=0,025)$. Entre as meninas observou-se que a preferência por achocolatados também reduziu significativamente após a intervenção (antes: 10,8\% e após: $3,7 \% ; p=0,038)$, assim como por sucrilhos (antes: $17,6 \%$ e após: $8,5 \% ; p=0,030$ ). Para os demais alimentos não foram encontradas diferenças significativas. (Tabela 4)

Em relação aos alimentos que, de acordo com a opinião dos escolares, deveriam ser oferecidos pelo PNAE (Tabela 4), observou-se a presença de hábitos diferenciados, com grande ocorrência de alimentos considerados inadequados à função exercida pelo programa. As frutas, entretanto, as quais não haviam aparecido na primeira coleta de dados entre as meninas, sobressaíram-se como o alimento mais citado pelas mesmas após a intervenção (antes: $0 \% \mathrm{e}$ após:15,2\%). Por outro lado, a menção a iogurtes diminuiu significativamente entre as garotas (antes: $20,0 \%$ e após: $3,0 \% ; p=0,001$ ).

\section{Discussão}

Pesquisas que utilizaram educação nutricional com crianças e adolescentes como uma das estratégias de intervenção relatam melhora nos conhecimentos nutricionais, atitudes e comportamento alimentar, influenciando também nos hábitos alimentares da família.25,26 Esses dados corroboram os resultados encontrados no presente estudo, onde se observou aumento da freqüência de algumas atitudes e práticas alimentares saudáveis após a realização da intervenção nutricional.

Porém, segundo Muller et al.,25 é improvável que intervenções isoladas em uma única área resolvam o problema da obesidade infantil. Aspectos como a influência dos pais, a pressão dos colegas, a publicidade e a auto-imagem, entre outros, devem ser considerados no desenvolvimento de estratégias para enfrentar a complexidade das causas.

No presente estudo, a realização da educação nutricional, como único meio de intervenção, não resultou em mudanças significativas no IMC dos escolares. Porém, é preciso considerar que o tempo entre a conclusão do programa educativo e a realização do segundo exame antropométrico (cerca de um mês) pode não ter sido suficiente para provocar alterações significativas nas medidas antropométricas dessa população.

O Programa Nacional de Alimentação Escolar (PNAE), por meio da distribuição de refeições durante o intervalo das atividades escolares, visa suplementar a alimentação dos alunos, melhorando as condições nutricionais e a capacidade de aprendizagem deles, além da formação de bons hábitos alimentares. 27 No entanto, a comercialização de alimentos, nem sempre saudáveis, pelas cantinas escolares pode interferir no consumo dos alimentos fornecidos pelo Programa. 13,28,29 Aqui, vale ressaltar que as mini-pizzas destacaram-se na escola pública como a preparação mais adquirida nas cantinas por escolares de ambos os sexos, mesmo após o desenvolvimento do programa educativo.

Alguns alimentos de baixo valor nutricional oferecidos nas cantinas são, muitas vezes, preferidos em detrimento de alimentos saudáveis, podendo contribuir para o consumo excessivo de gordura saturada e desenvolvimento de sobrepeso e obesidade. 28,30 A preferência dos escolares por refrigerantes também acaba tomando o lugar de bebidas mais nutritivas, como leite e derivados, ${ }^{29}$ fazendo com que a maioria das crianças não atinja a recomendação diária de cálcio. 28

Com a criação da Lei de Regulamentação das Cantinas ${ }^{31}$ em dezembro de 2001, ocorreram importantes mudanças na alimentação escolar. A comercialização de produtos considerados mais saudáveis tornou-se obrigatória, enquanto outros alimentos como salgadinhos industrializados, balas, pirulitos, refrigerantes e sucos artificiais tornaram-se expressamente proibidos. Porém, em algumas unidades escolares, alimentos inadequados continuam 
disponíveis. Deste modo, acredita-se ser de extrema importância o ensino sobre nutrição no ambiente escolar a fim de se promover hábitos alimentares saudáveis entre as crianças.

Analisando-se os resultados dessa pesquisa percebe-se ainda hábitos alimentares mais saudáveis entre os alunos de escola privada. Sabe-se que o nível socioeconômico interfere na prevalência de sobrepeso e obesidade, na medida em que determina a disponibilidade de alimentos e o acesso à informação. Estudando o consumo infantil de alimentos industrializados, Aquino e Philippi32 encontraram um alto consumo de açúcar em crianças com menor poder aquisitivo. Alguns estudos 33,34 explicam que, nos países desenvolvidos, a menor prevalência de obesidade infantil nas classes de renda elevada se deve ao maior acesso à informação acerca de padrões dietéticos e de atividade física mais saudáveis. O conhecimento dos pais sobre nutrição, suas influências sobre a escolha dos alimentos, padrões da alimentação em casa, seus níveis de atividade física e hábitos sedentários, como assistir televisão, são também determinantes no desenvolvimento dos hábitos das crianças. 6,31

\section{Referências}

1. WHO (World Health Organization). Obesity: preventing and managing the global epidemic. Geneva; 2000 (Technical Report Series, 894).

2. Nammi S, Koka S, Chinnala KM, Boini KM. Obesity: an overview on its current perspectives and treatment options. Nutr J. 2004; 3: (3). Available from: http://www.nutritionj. com/content/3/1/3 [2006 Jun 16]

3. Dehghan M, Akhtar-Danesh N, Merchant AT. Childhood obesity, prevalence and prevention. Nutr J. 2005; 4: (24) Available from: http://www.nutritionj.com/content/4/1/24 [2006 Jul 19]

4. Reilly JJ, Armstrong J, Dorosty AL, Emmett PM, Rogers I, Steer C, Sherriff A. Early life risk factors for obesity in childhood: cohort study. BMJ. 2005; 330: (1357). Available from: http://bmj.com/cgi/content/full/330/7504/1357 [2006 Jun 25]

5. Ronque ERV, Cyrino ES, Dórea VR, Júnior HS, Galdi EHG Arruda M. Prevalência de sobrepeso e obesidade em escolares de alto nível socioeconômico em Londrina, Paraná Brasil. Rev Nutr. 2005; 18: 709-17.

6. Silva GAP, Balaban G, Motta MEFA. Prevalência de sobrepeso e obesidade em crianças e adolescentes de diferentes condições socioeconômicas. Rev Bras Saúde Matern Infant. 2005; 5: 53-9.

7. Triches RM, Giugliani ERJ. Obesidade, práticas alimentares e conhecimentos de nutrição em escolares. Rev Saúde Pública. 2005; 39: 541-7.

8. Assis MA, Rolland-Cachera MF, Grosseman S, de Vasconcelos FA, Luna ME, Calvo MC, Barros MV, Pires MM, Bellisle F. Obesity, overweight and thinness in schoolchildren of the city of Florianopolis, Southern Brazil. Eur J Clin Nutr. 2005; 59: 1015-21.

9. Must A, Dallal GE, Dietz WH. Reference data for obesity: 85 th and 95 th percentiles of body mass index (wt/ht 2$)$ and triceps skinfold thickness. Am J Clin Nutr. 1991; 53: 83946.

10. Marins VMR, Almeida RMVRA, Pereira RA, Barros MBA Overweight and risk of overweight in schoolchildren in the city of Rio de Janeiro, Brasil: prevalence and characteristics. Ann Trop Pediatrics. 2002; 22: 137-44.
11. Kain J, Uauy R, Vio F, Albala C. Trends in overweight and obesity prevalence in Chilean children: comparison of three definitions. Eur J Nutr. 2002; 56: 200-4.

12. Wang Y, Monteiro CA, Popkin BM. Trends of obesity and underweight in older children and adolescents in the United States, Brazil, China, and Russia. Am J Clin Nutr. 2002; 75: 971-7.

13. Kain J, Olivares S, Castillo M, Vio F. Validación y aplicación de instrumentos para evaluar intervenciones educativas em obesidade de escolares. Rev Chil Pediatr. 2001; 72: $308-18$.

14. Briggs M, Safaii S, Beall DL. Position of the American Dietetic Association, Society for Nutrition Education, and American School Food Service Association - Nutrition services: an essential component of comprehensive school health programs. J Am Diet Assoc. 2003; 103: 505-14

15. Santos JS, Costa MCO, Nascimento Sobrinho CL, Silva MCM, Souza KEP, Melo BO. Perfil antropométrico e consumo alimentar de adolescentes de Teixeira de Freitas Bahia. Rev Nutr. 2005; 18: 623-32.

16. Sahota P, Rudolf MCJ, Dixey R, Hill AJ, Barth JH, Cade J. Evaluation of implementation and effect of primary school based intervention to reduce risk factors for obesity. BMJ. 2001; 323: 1-4.

17. ADA (American Dietetic Association). Reports: position of the American Dietetic Association, Society for Nutrition Education, and American School Food Service Association - Nutrition services: an essential component of comprehensive school health programs. J Am Diet Assoc. 2003; 103: 505-14.

18. Wechsler H, Deveraux RS, Davis M, Collins J. Using the school environment to promote physical activity and healthy eating. Prev Med. 2000; 31: 121-37.

19. Manios Y, Moschandreas J, Hatzis C, Kafatos A. Health and nutrition education in primary schools of Crete: changes in chronic disease risk factors following a 6-year intervention programme. Br J Nutr. 2002; 88: 315-24.

20. Bizzo MLG, Leder L. Educação nutricional nos parâmetros curriculares nacionais para o ensino fundamental. Rev Nutr. 2005; 18: 661-7. 
21. Boog MCF, Vieira CM, Oliveira NL, Fonseca O, L'Abbate S. Utilização de vídeo como estratégia de educação nutricional para adolescente: " comer... o fruto ou o produto?" Rev Nutr. 2003; 16: 281-93.

22. WHO (World Health Organization). Physical status: the use and interpretation of antropometry. Geneva; 1995 (Technical Reports Series 854).

23. CDC (Centers for Disease Control and Prevention). CDC growth charts: United States. [monography on line]. Atlanta: CDC; 2000. [cited 2002 may 6]. Available from: http:// www.cdc.gov/growthcharts [2002 May 6]

24. Philippi ST, Latterza AR, Cruz ATR, Ribeiro LC. Pirâmide alimentar adaptada: guia para escolha dos alimentos. Rev Nutr. 1999; 12: 65-80.

25. Muller MJ, Mast M, Asbeck I, Langnase K, Grund A. Preventions of obesity - it is possible? Obes Rev. 2001; 2: $15-28$.

26. Pérez-Rodrigo C, Aranceta J. School-based nutrition education: lessons learned and new perspectives. Publ Health Nutr. 2001; 4: 131-9.

27. Fundo Nacional de Desenvolvimento da educação: relatório de atividades. [monografia online] Brasília, DF: MEC; 2003. Acesso em 16 ago. 2006. Available from: http:// www.fnde.gov.br/Programas/ Merenda [2006 ago 16]

Recebido em 19 de setembro 2006

Versão final apresentada em 9 de outubro de 2007

Aprovado em 12 de dezembro de 2007
28. Mannino ML, Lee Y, Mitchell DC, Smiciklas-Wright H, Birch LL. The quality of girls'diets declines and tracks across middle childhood. Int J Behav Nutr Phys Act. 2004; 1: $1-11$.

29. Greves HM, Rivara FP. Report card on school snack food policies among the United States' largest school districts in 2004-2005: room for improvement. Int J Behav Nutr Phys Act. 2006; 3: 1-10.

30. Story M, Kaphingst KM, French S. The role of schools in obesity prevention. Future Child. 2006; 16: 109-42.

31. Santa Catarina. Lei n. ${ }^{\circ} 12.061$, de 18 de dezembro de 2001. Dispõe sobre critérios de concessão de serviços de lanches e bebidas nas unidades educacionais, localizadas no Estado de Santa Catarina. Lex: Diário Oficial do Estado de Santa Catarina; $200110 \mathrm{dez}$; p. 1

32. Aquino RC, Philippi ST. Consumo infantil de alimentos industrializados e renda familiar na cidade de São Paulo. Rev Saúde Pública. 2002; 36: 655-60.

33. Rolland-Cachera MF, Bellisle F. No correlation between adiposity and food intake: why are working class children fatter? Am J Clin Nutr. 1986; 44: 779-7.

34. Sunnegardh J, Bratteby LE, Hagman U, Samuelson G, Sjölin S. Physical activity in relation to energy intake and body fat in 8 and 13 year old children in Sweden. Acta Paediatr Scand. 1986; 75: 955-63. 